\title{
Need for Anaphoric Resolution towards Sentiment Analysis-A Case Study with Scarlet Pimpernel (Novel)
}

\author{
Dr.R.Nithya \\ Assistant Professor, Computer Science(Dept), Dr.N.G.P. Arts and Science College, Coimbatore, Tamil Nadu, \\ India
}

Received: 30 November 2018; Accepted: 17 December 2018; Published: 08 January 2019

\begin{abstract}
The problem of resolving references to earlier or later items in the discourse is commonly called as anaphora resolution or pronoun resolution. These items are usually noun phrases representing objects in the real world called referents but can also be verb phrases, whole sentences or paragraphs. Nowadays, anaphora resolution is addressed in numerous NLP (Natural Language Processing) applications. Proper treatment of anaphoric relations improves the performance of applications. Machine translation, information extraction, text summarization, or dialogue systems are some of the common applications of NLP. In early days, the machine translation systems processed on the basis of a sentence-by-sentence level. It did not consider the ties between sentences and resulted in an incoherent text as output. When the researcher forgets to handle the anaphora issue, it results in the striking problem of incorrect facts. It is very much needed to concentrate on the usage of pronoun, as it should match with their antecedents both in number and gender. Assigning inappropriate morphological features to the anaphor often may also lead to an undesirable change in the meaning of the sentence.
\end{abstract}

Index Terms: Sentiment Analysis, Anaphoric Resolution, Abstract Noun, Shell Noun.

(C) 2019 Published by MECS Publisher. Selection and/or peer review under responsibility of the Research Association of Modern Education and Computer Science.

\section{Introduction}

Social media is popularly known as democracy's pipeline, an amplifier of unfiltered emotion- is fundamentally creating a new shape. In fact, for many people accessing the Internet for the first time, social media was the reasons for people to access the Internet, "said the IAMAI-IMRB report on social media, 2016.

*Corresponding author Tel. No. 9790623017

E-mail:nithya.r.2018to2020@gmail.com 

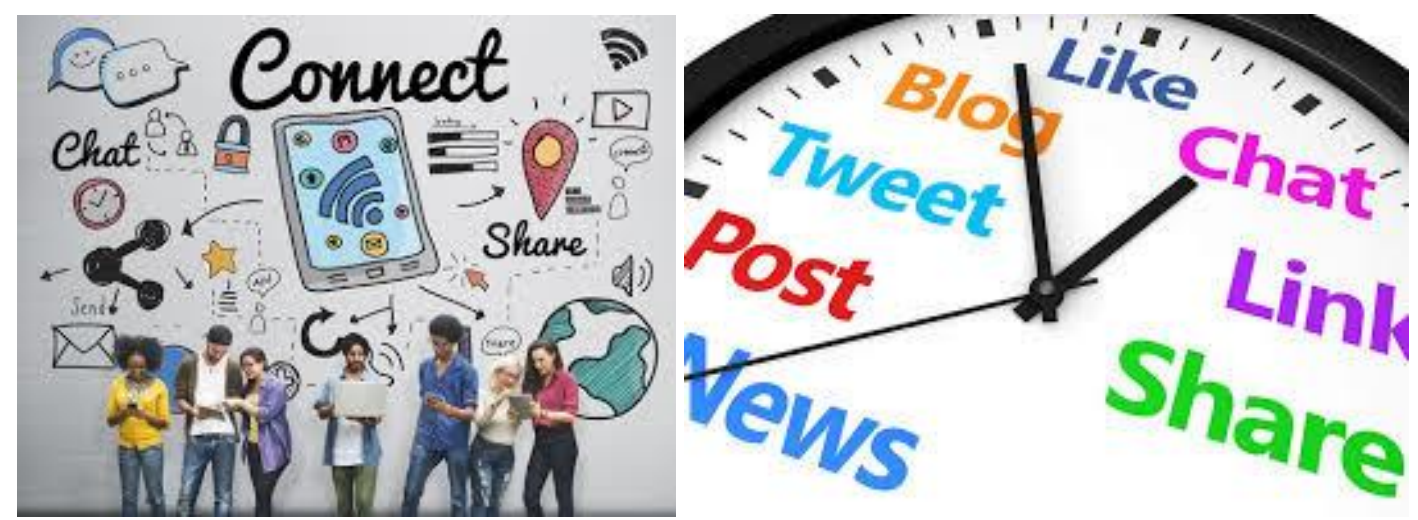

Fig.1. Social Media Trends

By 2018 it can either extend up to $\$ 12$ billion. Facebook and Twitter not only monitor users who are actively engaged in providing sentiment, opinion (attitude) but also add their unique insights to product penetration and reflect the changing moods of the public as shown in Fig 1.

\subsection{Emergence of $B I$}

Gartner, the world's leading information technology research and advisory company; predicted that huge change has occurred in the manner in which vendors' responses to the needs of their customers and they focused on standardizing the BI tools to handle vendor's needs, thus embracing new technology.Nowadays, most of the BI (Business Intelligence) components as shown in Fig 2. focuses on social media like Facebook, Twitter, blogs and online commercial websites like Shopclues, Pepperfry, Flipkart, Fabfurnish, TestFreaks, Amazon, Greendust etc. to gather comments posted by the sellers as shown in Fig 3.
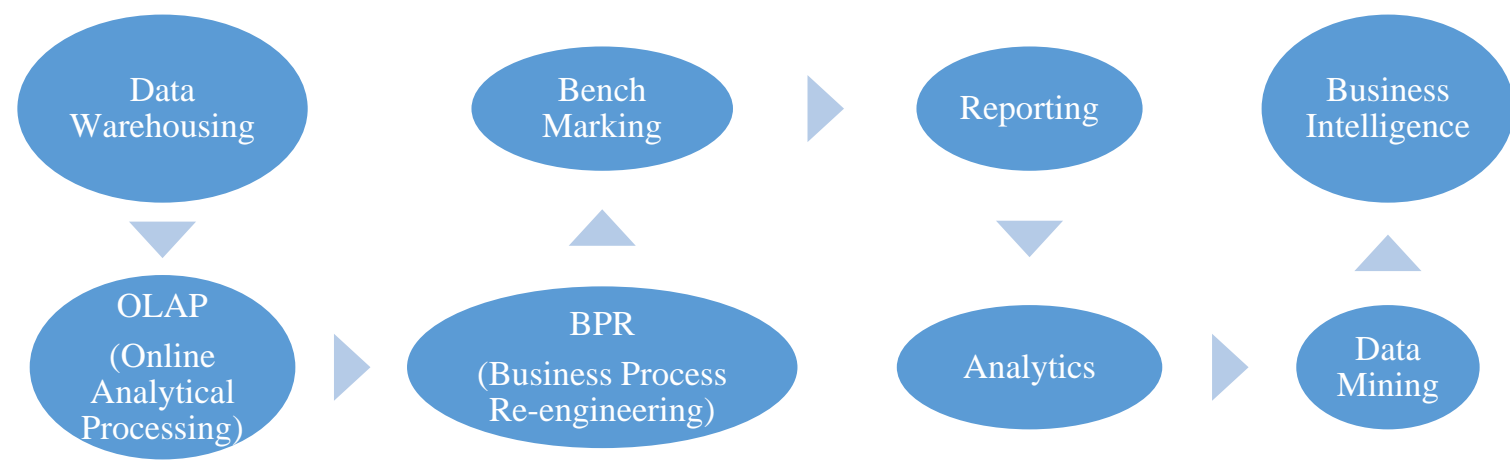

Fig.2. BI Components

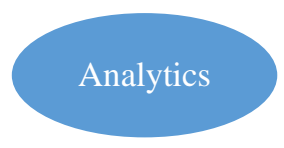

Data

Mining

In turn, the buyers were also willing to post their comment about each of the products they buy through online. 


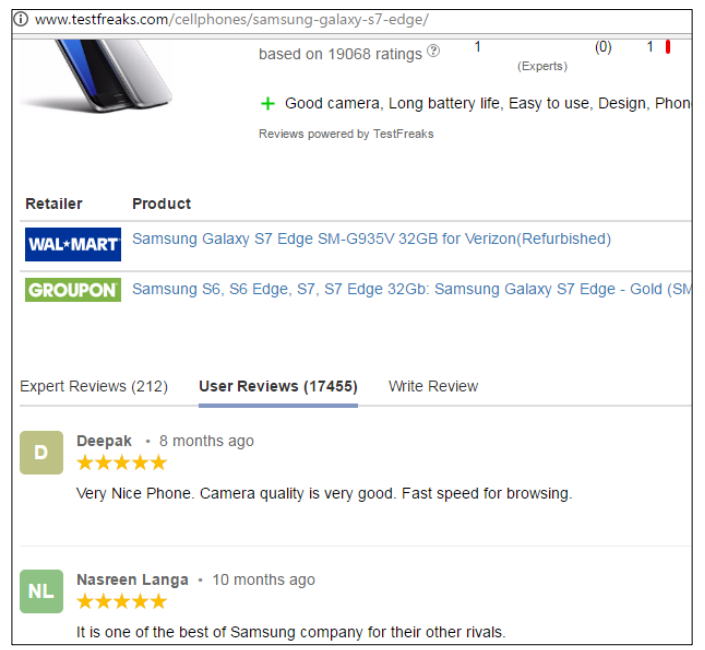

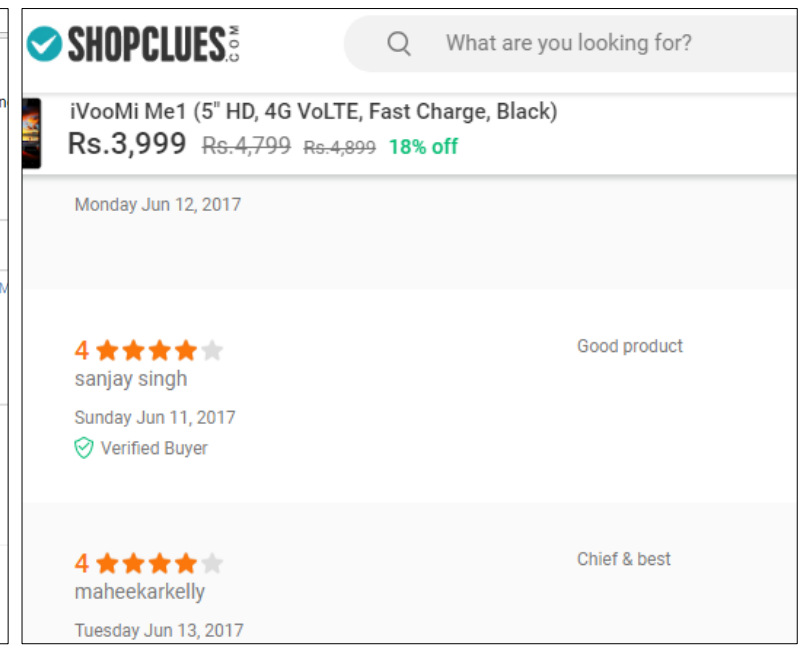

Fig.3. Examples of Short Reviews on Online Shopping Sites

These short reviews once refined and analyzed can help us to get a crystal clear idea about the buyers' view and also predict they will have on the buying decision of the future customers as shown in Fig 4.

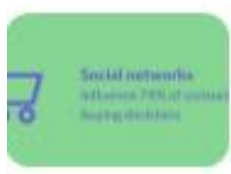

Demand Forecasting

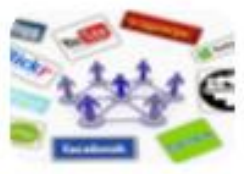

Brand promotion

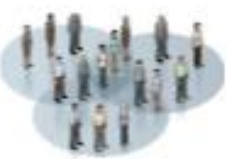

Market

Segmentation

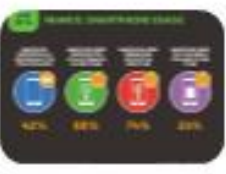

Product penetration

Fig.4. Typical Marketing Strategies

\subsection{Sentiment Analysis- A Bird's View}

In general, Opinion mining or Sentiment analysis is an important sub-discipline within data mining and NLP (Natural Language Processing), that deals with building a system that explores the users' opinions made on blog spot, comments, reviews, discussion, news, feedback or tweets, about a product, policy, person or a topic as shown in Fig 5. Bing Liu defines it in the following way:
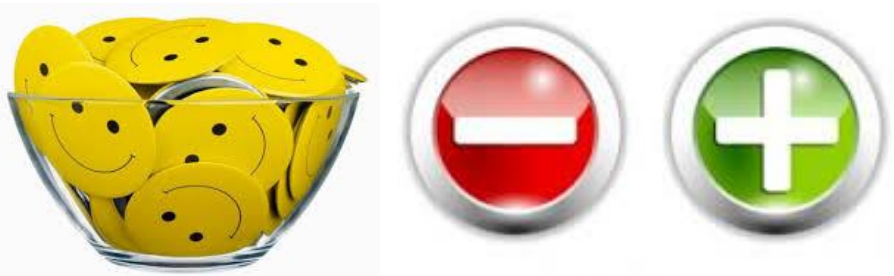

Fig.5. Sentiment Analysis Process 
"A Sentiment (or opinion) is a quadruple, $(\mathrm{g}, \mathrm{s}, \mathrm{h}, \mathrm{t}$ ), where $\mathrm{g}$ is the sentiment (or opinion) target, $\mathrm{s}$ is the sentiment about the target, $\mathrm{h}$ is the opinion holder and $\mathrm{t}$ is the time when the opinion was expressed."

To be specific, opinion mining can be defined as a sub-discipline of computational linguistics that focuses on extracting people's opinion from the web. It analyses the following from a given piece text about: -

Which part is opinion expressing?

What is being commented on?
Who wrote the opinion?

What is the opinion of the writer?

\subsection{WOM or eWOM}

Currently, 7\% of the products are sold due to the right handling of eWOM (electronic Word-Of-Mouth) or WOM (Word-Of-Mouth) strategy in the world market. This WOM act as a vessel carrying the brand of the product/policy launched in the market as shown in Fig 6.
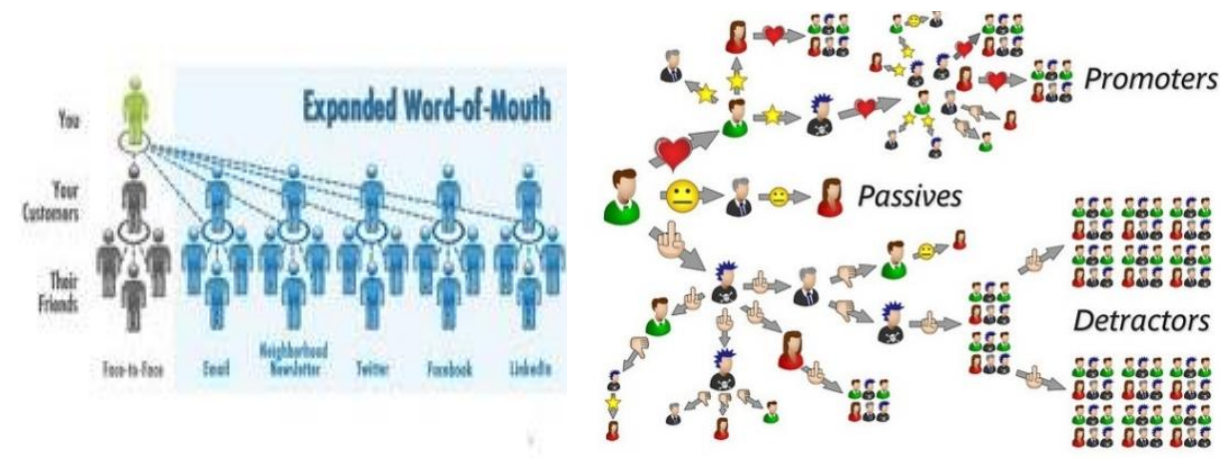

Fig.6. Word of Mouth Communication Patterns

Some of the viral videos that indicate the grooming of WOM strategies are listed below:-

- The Blender mixi which blends iPhone video has reached 10 million views thus making their gross profit reached 700 million.

- 'Rebecca black girl -Friday' acts as a right example for triggering out the right time for the product launch.

- 'Never Say No to Panda Cheese' is a viral video that creates more curiosity among the public as the brand name 'Panda' cannot be avoided when people discuss stories about the quality of cheese they consume.

- McDonald's Locator App - Using eWOM, one technical person decided to build an app which decides about where to locate the McDonald in a customer's city.

\subsection{Sentiment Analysis- an Evolution}

SA (Sentiment Analysis) evolves with different dimensional subtasks as shown in Fig 7. Among them, the first three dimensions of subtask are highly in demand due to their role in creating more business opportunities/intelligent system. 


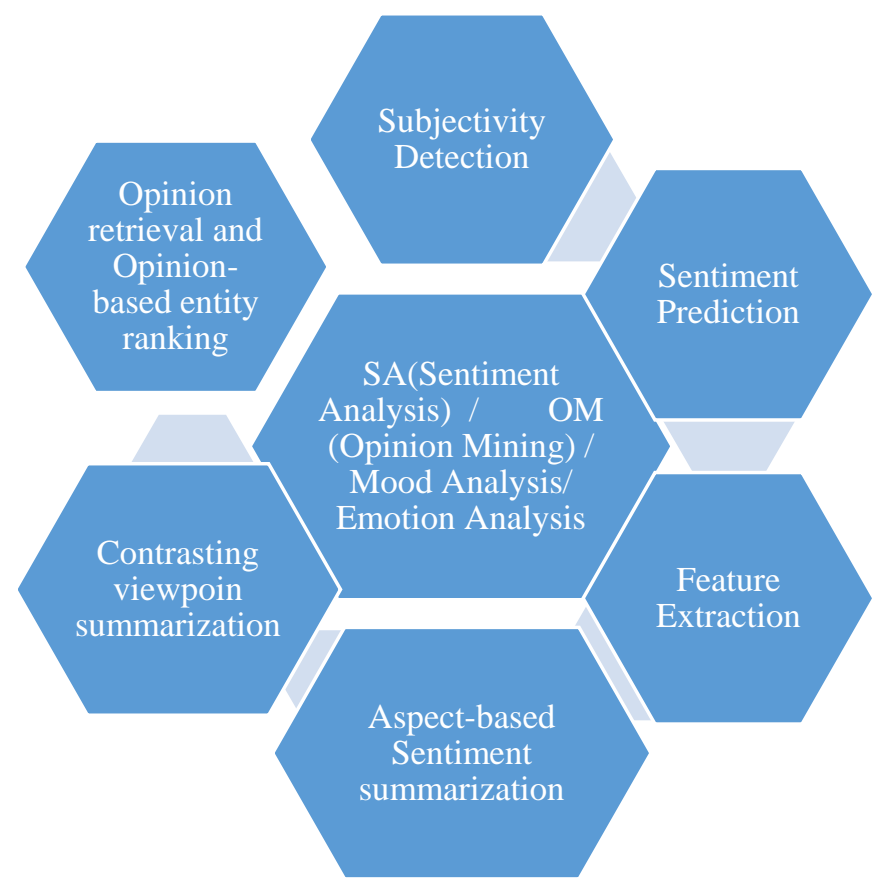

Fig.7. Various Sub-dimensional tasks of Sentiment Analysis

\subsection{Applications}

A few instances where Sentiment Analysis played a major role in India are,

- Economic and Political risk of India's demonetization.

- Government Schemes like Make in India, Digital India, Smart Cities, Atal Pension, Swachh Bharat Abhiyan, Pradhan Mantri Suraksha Bima Yojana, Jan Dhan Yojana, etc.

- Pradhan Mantri Gram Parivahan Yojana (PMGPY) - Interest Free Commercial Vehicle Loan for Women.

- Use of digital payment mode (or Cashless System)in India like E-Wallets- PayTM, Plastic Money Debit/Credit cards, UPI - Unified payments Interface Apps,Net Banking - Online Fund Transfer, Aadhaar Card - Aadhaar Enabled Payment System.

- Launch of Reliance Jio Sim to meet the customer needs- Brand Promotion shown in Fig 8.

- The voice of Jallikattu showed in Fig 9.

- Boycott of Pepsi-Coke in Tamil Nadu.

- Abolishing the NEET entrance test for medical admissions in Tamil Nadu.

- Promoting new strategies on GST (Goods and Services Tax) - to get rid of tax terrorism and helping to curb black money and corruption.

- "Save Neduvasal, Save Thamirabarani River and Ban Tasmac" are the slogans raised by the public in and around the cities in Tamil Nadu.

- Banning water-depleting Prosopis Juliflora tree (Semai Karuvelam Maram) in and around the cities in Tamil Nadu. 


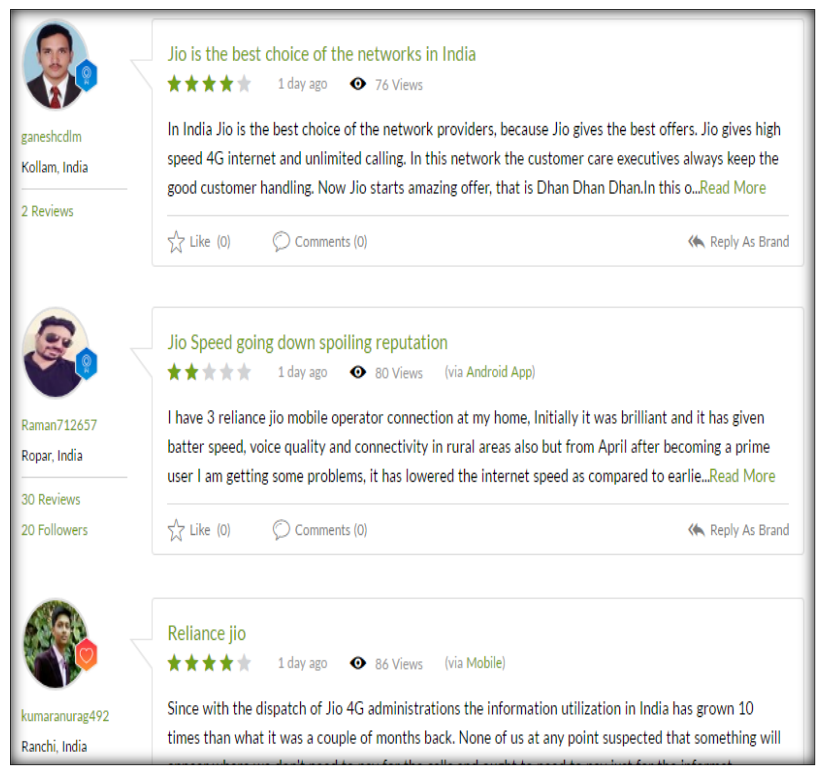

Fig.8. Public Opinion on Launch of Jio Sim at Mouthshut.com

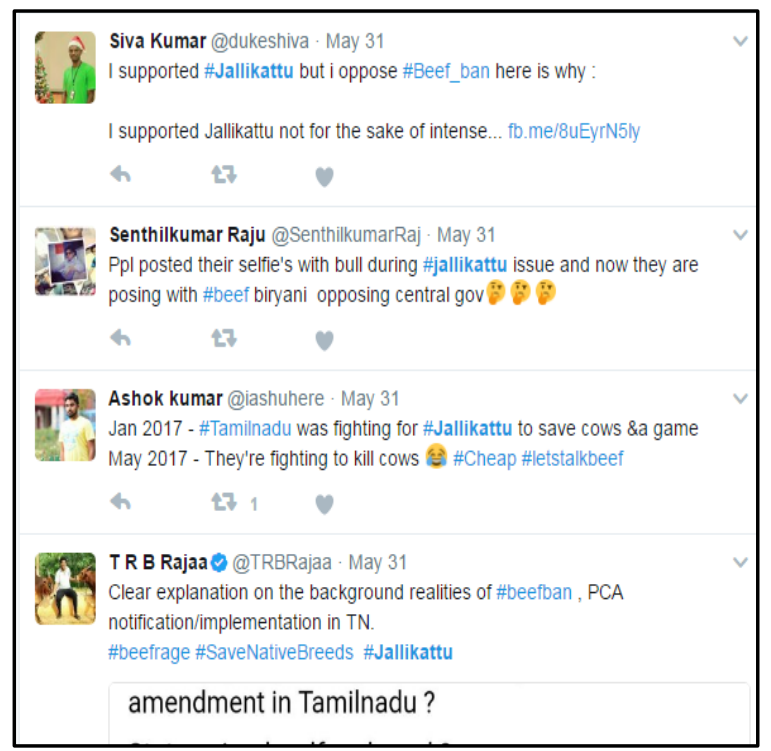

Fig.9. Public Opinion about Jallikattu at Twitter

Therefore, SA enables the people to closely watch each move taken by politicians, celebrities, businessmen and spiritual personalities. In particular, it acts as a democratic watchdog in sports, technology, medicine, space research, astrology, education and what not. 


\section{Related Works}

Sentiment analysis for the emotional preference of online comments has gained great achievement since it was raised up by Pang et al. and studied in-depth. Hu and Liu et al attempt to find features by performing POS tagging and generate n-grams, for sentiment prediction. Conventionally, the anaphoric resolution depends mainly on syntactic, semantic or pragmatic clues to in order to identify the preliminary of an anaphor. Hobbs' was the foremost researcher to show his interest on this particular domain. He has extracted part of syntactic tree that helps in checking the gender of antecedent candidates and a specified pronoun. Lappin and Leass proposed the Resolution of Anaphora Procedure which endures on applying the syntactic representations generated by the parser framed by McCord's Slot. It also reckon to intrusive measures deserted from syntactic structure. The system does not include any real world facts or semantic meaning for deciding the candidates. An advanced version of RAP was proposed by Kennedy and Boguraev which mainly confide in POS tagging with shallow parsing. It shows there is something lagging on parsing adjunct noun phrases. Cardie et al.,decided to proceed with co-reference as a clustering task. Then he also applied a distance metric function to calculate the similarity between the two noun phrases. Denber, proposed an algorithm called Anaphora Matcher (AM) which handles inter-sentential anaphora over a two-sentence context. This algorithm proclaims on both the real world knowledge and semantic meanings. Inorder to get the clues of semantic understanding the lexical database named WordNet was used. Denber also affirmed that most anaphora does not refer back more than one sentence in any case. Dagan and Itai exclaimed the statistical approach as an alternate way to disambiguate pronouns using corpus information, which mainly depends on constraints knowledge. They examined the pronoun "it" randomly inorder to resolve it randomly. Their model also focus on co-occurrence patterns mainly obtained from the corpus to identify the antecedents. It results with the selection of the highest frequency in the co-occurence patterns as an antecedent candidate that exactly match to the anaphor. A knowledge-poor approach is focused by Mitkov, and that can be applied to any different languages like English, Polish, and Arabic. Antecedent indicator is mainly used for assignment of scores as 2, 1, 0,-1 against each of candidate noun phrase. Thus they play a vital role in identifying the antecedents from a defined set of candidates. Another system named CogNIAC was developed by Baldwin inorder to resolve the anaphoric resolution with limited knowledge and linguistic resources. It is capable of attaining $90 \%$ precision with $60 \%$ recall. Thus he conferred a more dynamic and revitalized version of Mitkov's knowledge-poor approach to pronoun resolution. To the contrast of previous anaphoric resolution the brand new MARS, operates completely in an automated mode. The Boost Pronoun, Syntactic Parallelism and Frequent Candidates were the three new factors of indication used in MARS. Mitkov recommended an evaluating platform for comparing anaphora resolution algorithms which is demonstrated by presenting the results of the comparative evaluation on the criteria of several accuracy metrics. Their testing phase mainly includes 362 anaphoric expressions, 422 pronoun, 19305 nouns out of 28,272 words. The progress rate calculated for the 422 pronouns found in the texts was $56.9 \%$ for Mitkov's method, $49.72 \%$ for Cogniac and $61.6 \%$ for Kennedy and Boguraev's method.

\section{A Simple Anaphoric Resolution}

Need: Today, consumers make buying decisions on the customer review that is available on every online shopping sites. Nowadays, many websites have been developed which emphasise on the participation. For example, Amazon.com encourages people to write their opinion about the products and discusses the features of that product. Online shoppers often have different ideas about the same product. They look for the product features that are consistent with their goal. Gathering these reviews helps manufacturers to be aware of the strength and weakness of their product and further improve it. But it is not easy to explore a lot of reviews and read the comments carefully in order to find which attribute or component of the product has received more feedbacks from the consumers. The problem of resolving references to earlier or later items in the discourse is commonly called as anaphora resolution or pronoun resolution. These items are usually noun phrases 
representing objects in the real world called referents but can also be verb phrases, whole sentences or paragraphs.

\section{1. $\operatorname{Noun}(N N)$ :}

In traditional grammar, a noun (is a part of speech) that denotes a person, animal, place, thing at common. There are various types of methods to classify the types of nouns that exist in the English grammar.

\subsection{Classifications of Noun(s)}

Proper Noun, Common Noun, Pronouns,Compound Noun,Countable Noun,Uncountable Noun, Concrete Noun, Collective Noun and Abstract Noun.

\subsubsection{Pronoun:}

In English grammar, the personal pronouns are types of nouns that take the place of nouns when referring to people, places or things. For example, I, you, he, she, it, and they are the pronouns.

Raj works at a work shop. He works at a work shop.

These pronouns take on other forms depending on what type of function they are performing in a sentence. When used to signify possession of another noun, pronouns take on their possessive form such as mine, ours, hers, and theirs.

That coffee shop belongs to Mercy. That shop is hers.

In the above sentence, look at the second part "That shop is hers". Here "shop" is the subjective part and "hers" is the predicate or objective part. The objective part of the preposition is usually followed by the pronoun like him, her, us, me and them.

\subsection{Scope for Conducting on Resolving Anaphora(s)}

To undergo base work on resolving the anaphoric conflict, a part of the text from the novel, 'The Scarlet Pimpernel' is taken as dataset in .txt file as shown in Fig 10.

But they were nearly always caught at the
barricades, Sergeant Bibot especially at the
West Gate had a wonderful nose for scenting
an aristo in the most perfect disguise. Then, of
course, the fun began. Bibot would look at his
prey as a cat looks upon the mouse, play with
him, sometimes for quite a quarter of an hour,
pretend to be hoodwinked by the disguise, by
the wigs and other bits of theatrical make-up
which hid the identity of a CI-DEVANT noble
marquise or count.

Fig.10. Storyline from the novel 'The Scarlet Pimpernel' 


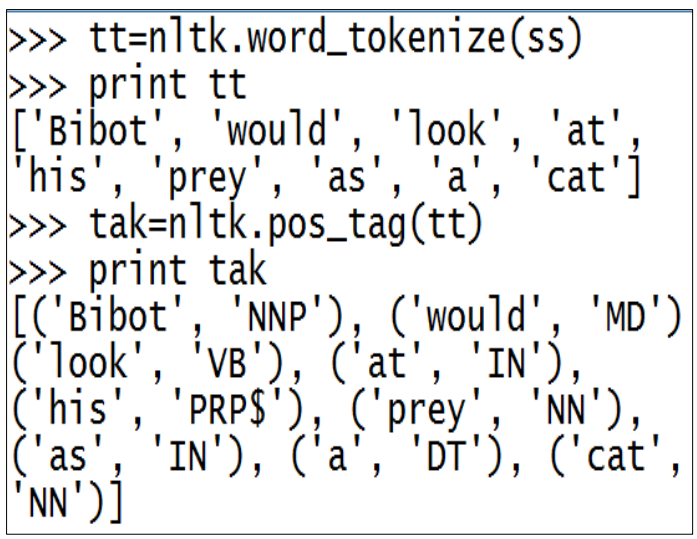

Fig.11. Tokenization and Parsing

The text highlighted includes the proper noun 'Bibot' and pronoun 'his'. When this sentence is tokenized and parsed it results with the POS as stated below in Fig 11.

Most of the researchers have contributed their work in analyzing the sentiment behind the text by concentrating at most on noun and adjective combinations. And currently the researchers are turning the focus on the adverb and prepositions that occur along with base patterns too. But there are only very few studies made on pronoun and abstract noun and its significant role in bearing sentiment.

When a pronoun is replaced by its antecedent noun term, it can surely improve the classification accuracy. With this idea of anaphoric resolution, a demo work is made over here.

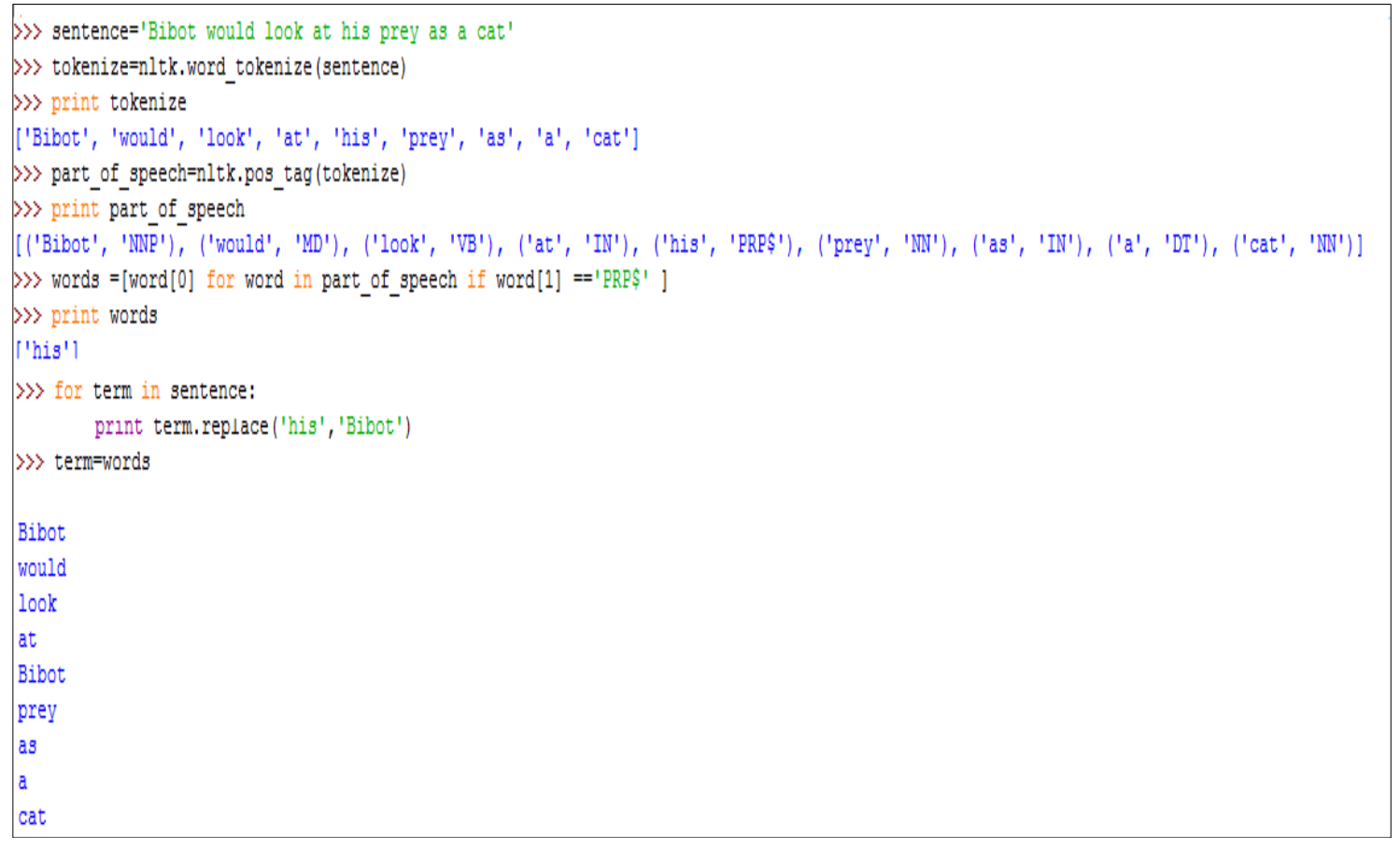

Fig.12. Replacement of Pronoun by Proper Noun - Resolving Anaphora 
Here, the Figure 12. reveals that the pronoun gets replaced by the antecedent common noun using Python code.But this is not a simple task, as it needs a lot of sub-dimensional task to resolve specific anaphora(s) like his, her, this etc. Each of the sentences have to be keenly examined for identifying the pronoun and its antecedents for resolving it.

\subsection{Abstract Noun}

Abstract nouns have no physical existence and they are used to refer to theoretical concepts like ideas, emotions, and concepts that cannot be seen, touched, heard, smelled, or tasted. Example: redemption, love, time, fear, freedom, hate, power.The Noun form of adjectives or verbs is actually called as an abstract noun.

Example: All you need is love. We must fight the power.

In this sentence, the term love and power are intangible or non-measurable feeling/emotion that cannot be concretely perceived are referred. The next more specific scope for anaphora resolution is of identifying the abstract or shell noun from the given dataset.

\subsubsection{Shell Noun}

According to cognitive linguistics, a shell noun is an abstract noun that particularly refers to a complex idea. A shell noun cannot be identified on the basis of its inherent lexical meaning but then on the basis of its behavior in an individual clause. It concentrates on actions, events, ideas, states of mind, qualities and conditions. The following table.1 shows different types of shell nouns. Certain abstract or shell nouns, especially the ones describing feelings and emotions, easily fit into multiple categories, as they can be used in different places in different ways.

Table 1. Different Types of Shell Noun

\begin{tabular}{|c|c|c|c|c|c|c|}
\hline Feelings & States & Emotions & Qualities & Concepts & Ideas & Events \\
\hline Anxiety & Being & Anger & Beauty & charity & Beliefs & adventure \\
\hline Confusion & Chaos & Despair & Beauty & comfort & Communication & Birthday \\
\hline Fear & Freedom & happiness & Brilliance & culture & Curiosity & Career \\
\hline Pain & Liberty & Hate & Courage & deceit & democracy & childhood \\
\hline Pleasure & Luxury & Indifference & Dedication & energy & friendship & Death \\
\hline Satisfaction & Misery & Joy & Determination & failure & Interest & Future \\
\hline sensitivity & Nervousness & Grief & Generosity & faith & knowledge & Holiday \\
\hline Stress & Openness & Love & Honesty & Motivation & Thought & Life \\
\hline Sympathy & Peace & Sadness & Patience & opportunity & Sacrifice & Marriage \\
\hline Warmth & Pessimism & Sorrow & Trust & perseverance & Wisdom & Past \\
\hline
\end{tabular}


The same Scarlet Pimpernel novel is taken for identifying the abstract noun and for further mapping to its family.

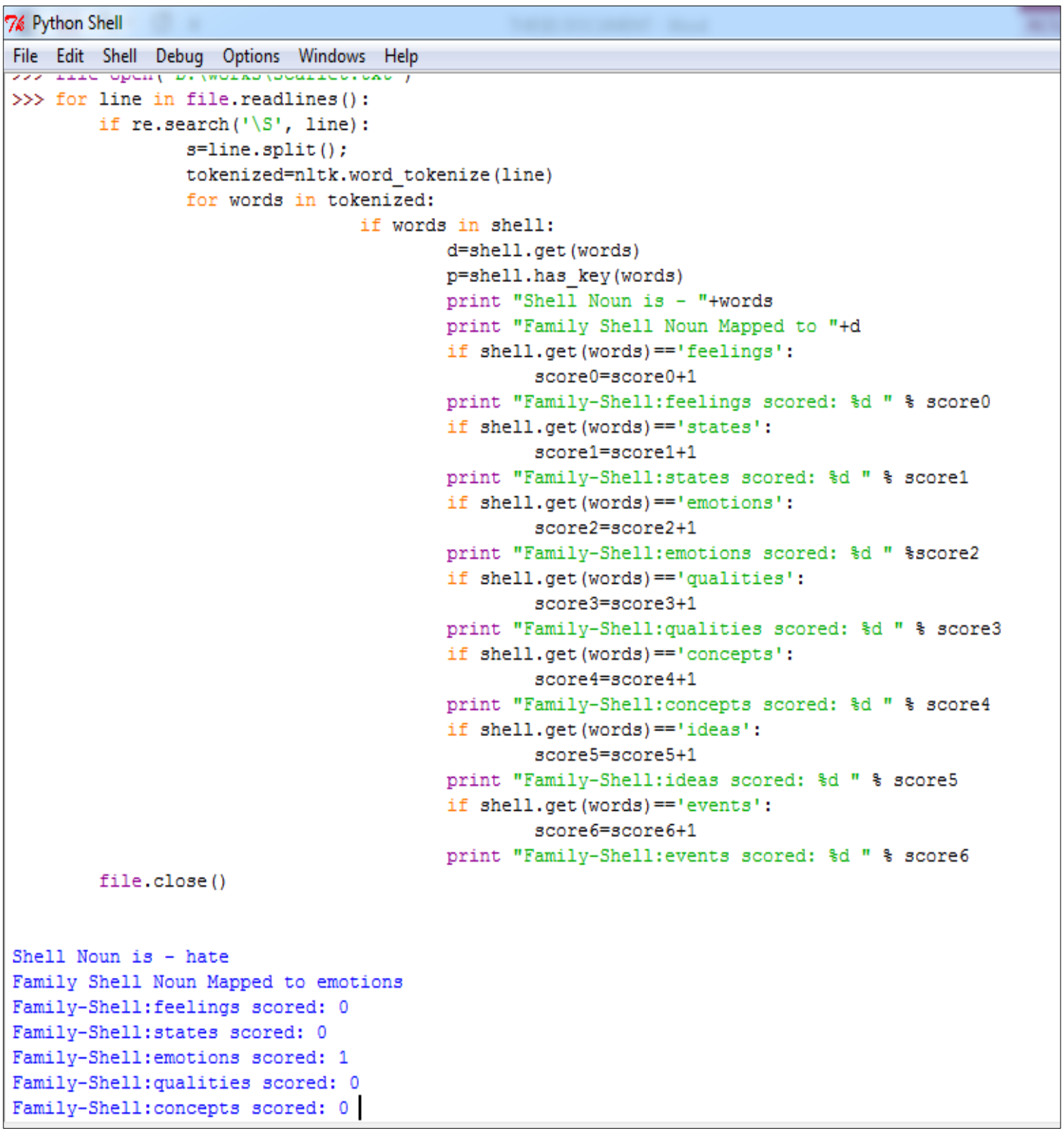

Fig.13. Calculating the Family-shell Mapping Score

Here a lexicon dictionary is built for seven specific family shells like feelings, states, emotions, qualities, concepts, ideas and events. Each abstract noun is mapped towards these specific family- shell. Once the algorithm is executed as shown in Fig 13., it searches for abstract nouns in each of the sentences and finally 
displays its score as shown in Fig 14. With this result, it is possible to arrive at a research scope that these abstract nouns are to be handled carefully while undergoing sentiment analysis.

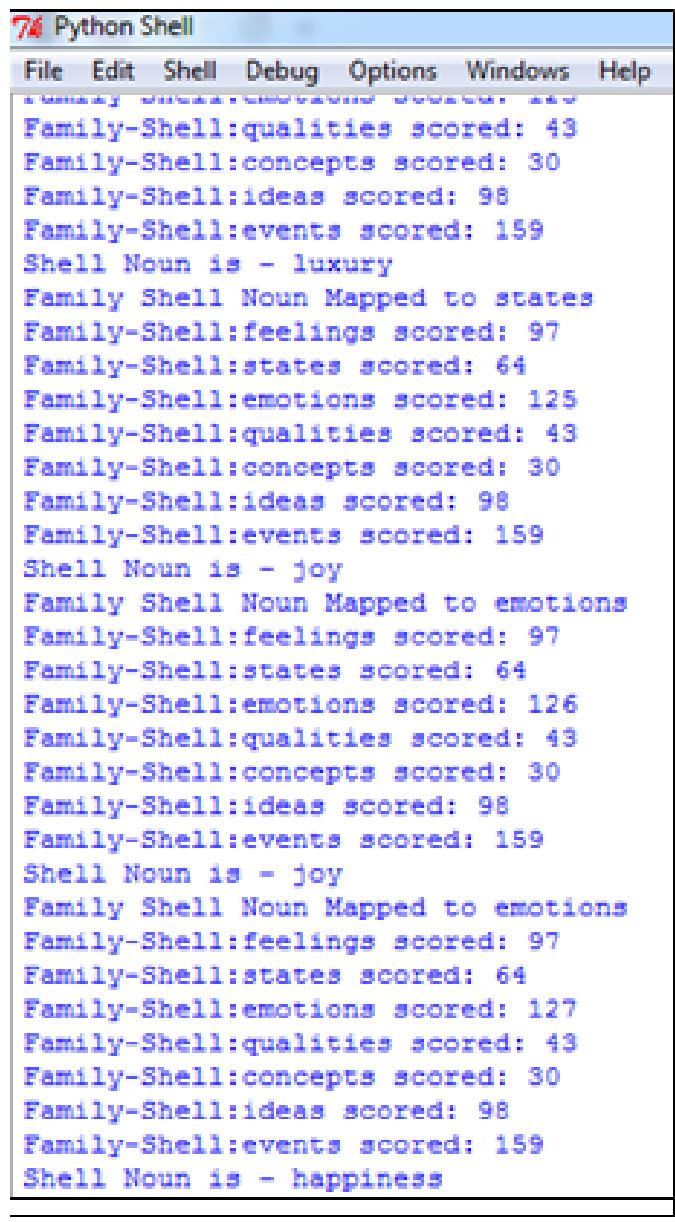

Fig.14. Final score- Abstract Noun Mapped to Family-shell

Table 2. Shell noun map to Family-shell count and its Frequency of Occurrence

\begin{tabular}{|l|l|}
\hline Shell nouns & Frequency of occurrence \\
Feelings & 97 \\
States & 64 \\
Emotions & 128 \\
\hline Qualities & 43 \\
\hline Concepts & 30 \\
\hline Ideas & 98 \\
\hline Events & 159 \\
\hline
\end{tabular}




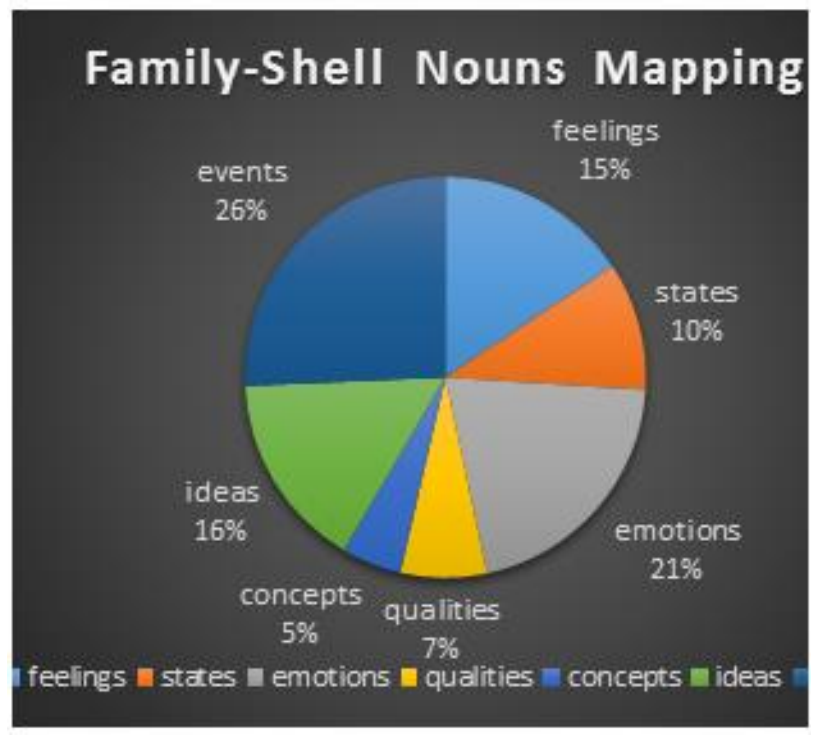

Fig.15. Family-Shell Nouns Mapping Distribution

From the Fig 15. it is clearly understood that an abstract noun words that are mapped towards the family of feelings (yielding 15\%) and emotions (yielding 21\%) and the patterns that include these abstract nouns have to be highly concentrated for analyzing the sentiment. In that (The Scarlet Pimpernel) novel, the writer has contributed altogether of $36 \%$ on emotions and feelings. And these abstract nouns are to be carefully handled when the analysis is to be made particularly towards analyzing the best characters.

\section{Conclusion}

Thus this paper finally reveals the importance of performing anaphoric resolution with a simple proposal. It is a wide research area, and once the resolution is made it can predominantly improve the accuracy of undergoing sentiment analysis. This paper also showcases the noteworthiness of identifying the shell nouns. Therefore the significance of resolving the shell noun is clearly visualized and demonstrated using FamilyShell mapping. They are the greatest source of sentiment bearing factor. Once this resolution is made it can positively improve the accuracy of the system.

\section{References}

[1] Aarts Jan, Henk Barkema and Nelleke Oostdijk (1997), “The TOSCA-ICLE Tagset: Tagging Manual”, TOSCA Research Group for Corpus Linguistics.

[2] Baldwin, Breck (1997), "CogNIAC: high precision coreference with limited knowledge and linguistic resources", Proceedings of the ACL'97/EACL'97 workshop on Operational factors in practical, robust anaphora resolution, pp. 38-45.

[3] Bontcheva, Kalina, Marin Dimitrov, Diana Maynard and Valentin Tablan (2002), "Shallow Methods for Named Entity Coreference Resolution", Proceedings of TRAITEMENT AUTOMATIQUE DES LANGUES NATURELLES (TALN), pp. 24-32.

[4] Cardie, Claire and Kiri Wagstaff (1999), "Noun Phrase Coreference as Clustering", Proceedings of the Joint Conference on Empirical Methods in Natural Language Processing and Very Large Corpora. 
[5] Chen, Kuang-hua and Hsin-Hsi Chen (1994), "Extracting Noun Phrases from Large-Scale Texts: A Hybrid Approach and Its Automatic Evaluation", Proceedings of the 32nd ACL Annual Meeting, 1994, pp. 234-241.

[6] Dagan, Ido and Alon Itai (1990), "Automatic processing of large corpora for the resolution of anaphora references", Proceedings of the 13th International Conference on Computational Linguistics (COLING'90), Vol. III, 1-3, Helsinki, Finland.

[7] Denber, Michel (1998), "Automatic resolution of anaphora in English", Technical report, Eastman Kodak Co. Evans, Richard and Constantin Orasan (2000), "Improving anaphora resolution by identifying animate entities in texts", In Proceedings of DAARC-2000.

[8] Ge, Niyu, John Hale and Eugene Charniak (1998), "A Statistical Approach to Anaphora Resolution", Proceedings of the Sixth Workshop on Very Large Corpora (COLING-ACL98), pp.161-170.

[9] Kennedy, Christopher and Branimir Boguraev (1996), "Anaphora for everyone: Pronominal anaphora resolution without a parser", Proceedings of the 16th International Conference on Computational Linguistics, pp.113-118.

[10] Lappin, Shalom and Herbert Leass (1994), "An Algorithm for Pronominal Anaphora Resolution", Computational Linguistics, Volume 20, Part 4, pp. 535-561.

[11] Miller, George (1993), "Nouns in WordNet: A Lexical Inheritance System", Journal of Lexicography, pp. 245-264.

[12] Mitkov, Ruslan (1998), "Robust pronoun resolution with limited knowledge", Proceedings of the 18th International Conference on Computational Linguistics (COLING'98)/ACL'98 Conference Montreal, Canada. pp. 869-875. 17

[13] Mitkov, Ruslan (1999), "Anaphora Resolution: The State of the Art”, Working paper (Based on the COLING'98/ACL'98 tutorial on anaphora resolution)

[14] Mitkov, Ruslan and Catalina Barbu (2001), "Evaluation tool for rule-based anaphora resolution methods", Proceedings of ACL'01, Toulouse, 2001.

[15] Mitkov, Ruslan, Richard Evans and Constantin Orasan (2002), "A new, fully automatic version of Mitkov's knowledge-poor pronoun resolution method", In Proceedings of CICLing- 2000, Mexico City, Mexico.

[16] Wang, Ning, Chunfa Yuan, K.F. Wang and Wenjie Li (2002), "Anaphora Resolution in Chinese Financial News for Information Extraction", Proceedings of 4th World Congress on Intelligent Control and Automation, June 2002, Shanghai, pp.2422-2426.

\section{Authors' Profile}

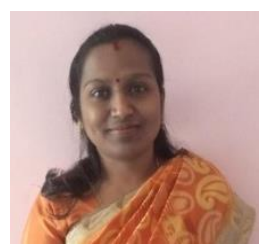

Nithya Ramachandran working as assistant professor in profound institution and is keenly interested on web designing frameworks and python programming. She has also completed her Ph.D in computer science by exploring various NLP techniques for analyzing sentiments behind the customer reviews. She also shows her interest in inferring discrete mathematics rules on designing mathematical formulations.

How to cite this paper: R.Nithya,"Need for Anaphoric Resolution towards Sentiment Analysis-A Case Study with Scarlet Pimpernel (Novel)", International Journal of Education and Management Engineering(IJEME), Vol.9, No.1, pp.37-50, 2019.DOI: 10.5815/ijeme.2019.01.04 